\title{
Macroinvertebrados como Bioindicadores da Qualidade da Água do Rio Paraíba do Sul, Barra Mansa, Rio de Janeiro, Brasil
}

\author{
Macroinvertebrados como Bioindicadores da Qualidade da Água do \\ Rio Paraiba do Sul, Barra Mansa, Rio de Janeiro, Brasil
}

\author{
Macroinvertebrates as Bioindicators of the Water Quality of the \\ Paraíba do Sul River, Barra Mansa, Rio de Janeiro, Brazil
}

Os ecossistemas aquáticos estão sujeitos a vários tipos de perturbações antrópicas que alteram a estrutura e o funcionamento destes ambientes. Para avaliar tais mudanças pode- se utilizar o biomonitoramento, que é uma ferramenta importante para determinar a qualidade da água através do uso sistemático de respostas biológicas inferidas pela presença e abundância de organismos bioindicadores. Organismos bentônicos têm sido amplamente utilizados como indicadores de qualidade ambiental, pois são sensíveis a vários tipos de degradação, e respondem diferentemente a um amplo espectro de níveis e tipos de poluição. $O$ objetivo deste trabalho foi realizar o levantamento de macroinvertebrados no Rio Paraíba do Sul em Barra Mansa (Rio de Janeiro), para averiguar os impactos ambientais causados pela intervenção do homem ao meio, causando seu desequilibrio. Realizaram-se coletas em 7 pontos ao longo da margem direita do Rio utilizando-se um puçá, pelo método de arraste. Seguiu-se a esta etapa, a lavagem, flutuação, triagem e identificação dos macroinvertebrados com literatura especifica. Foram identificados 211 organismos, pertencentes a 11 famílias, distribuídos nos filos Mollusca, Annelida e Arthropoda. Foi calculado o índice Biological Monitoring Working Party System (BMWP) para determinar a tolerância dos organismos encontrados quanto à poluição orgânica. O ponto 4 apresentou maior abundância de organismos e a classe Gastropoda foi a mais dominante. Todos os pontos apresentaram representantes $d a$ família Physidae. Houve uma baixa diversidade de famílias, com poucas diferenças entre elas.
The aquatic ecosystems are subject to several types of disturbances anthropics that alter the structure and the function of those environments. To assess these changes can be utilized biomonitoring, which is an important tool to determine water quality through the systematic use of biological responses inferred by the presence and abundance of organisms bioindicators. Organisms benthic have been used thoroughly as indicators of environmental quality, because they are sensitive to several types of environmental degradation and they answer differently to a wide level spectrum and pollution types. The aim of this study was the survey of macroinvertebrates in Rio Paraiba do Sul in Barra Mansa (Rio de Janeiro), to investigate the environmental impacts caused by the intervention of man in half, causing their imbalance. Collections were carried out at 7 points along the right bank of the river using a dip net, by the method of drag. This was followed by this step, washing, flotation, screening and identification of macroinvertebrates in the literature specific. 211 organisms, belonging to 11 families, distributed in the phyla Mollusca, Annelida and Arthropoda were identified. The index Biological Monitoring System Working Party (BMWP) was calculated to determine the tolerance of organisms found as the organic pollution. Point 4 showed a higher abundance of organisms and the Gastropoda class was the most dominant. All points presented representatives of the family Physidae. There was a low diversity of families, with very few differences between them.
Los ecosistemas acuáticos están sujetos a varios tipos de trastornos anthrophics que alteran la estructura y la función de esos entornos. Para evaluar estos cambios pueden ser utilizados biomonitoreo, que es una herramienta importante para determinar la calidad del agua a través de la utilización sistemática de respuestas biológicas inferidos por la presencia y abundancia de organismos bioindicadores. Organismos bentónicos se han utilizado a fondo como indicadores de la calidad del medio ambiente, porque son sensibles a varios tipos de degradación del medio ambiente y responden de manera diferente a una amplia tipos de espectro nivel y la contaminación. El objetivo de este trabajo fue el estudio de macroinvertebrados en Río Paraíba do Sul en Barra Mansa (Río de Janeiro), para investigar los impactos ambientales causados por la intervención del hombre en el medio, la causa de su desequilibrio. Colecciones se realizaron a 7 puntos a lo largo de la margen derecha del río utilizando una red de inmersión, por el método de arrastre. Esto fue seguido por este paso, lavado, flotación, la detección y la identificación de macroinvertebrados en la literatura específica. Se identificaron 211 organismos, pertenecientes a 11 familias, distribuidas en los filos moluscos, anélidos y artrópodos. El índice de Monitoreo Biológico Grupo de Trabajo del Sistema (BMWP) se calculó para determinar la tolerancia de los organismos que se encuentran como la contaminación orgánica. Punto 4 mostró una mayor abundancia de organismos y la clase Gastropoda fue el más dominante. Todos los puntos presentados representantes de la familia Physidae. Hubo una baja diversidad de familias, con muy pocas diferencias entre ellos. 
Palavras-chave: Barra Mansa; Bioindicadores; Macroinvertebrados; Rio Paraiba do Sul.zqqes

\section{Autores: \\ Roney Rodrigues Guimarães}

Doutor em Biologia Animal pela Universidade Federal Rural do Rio de Janeiro (UFRRJ). Graduação em Ciências Biológicas e Graduação Tecnológica em Gestão Ambiental (Cursando), Especialização em Biologia Parasitária, Mestrado em Ciências Biológicas (Doenças Parasitárias) e Docente da Universidade Estácio de Sá (UNESA - Presencial e EAD) e do Centro Universitário de Barra Mansa (UBM). Editor Cientifico e Consultor Adhoc do Periódico "On Line" EntomoBrasilis. Assessor Ad-hoc - Fundação de Amparo à Pesquisa do Estado de São Paulo (FAPESP). Membro do Conselho Editorial Externo da Revista Cientifica do Centro Universitário de Barra Mansa. INEP - Avaliador do BASIs nos Instrumentos de Autorização, Reconhecimento e Renovação de Reconhecimento de Cursos. Diretor de Projetos do Centro de Educação e Pesquisas em Medicina Ambiental. (CEMA).

E-mail: roneyrg@gmail.com

\section{Lidiane Fontes de Carvalho Silva}

Bacharel em Biologia pelo Centro Universitário de Barra Mansa. Estagiária no Programa de Estudo, Manejo e Conservação do Bicho Preguiça no Parque Centenário de Barra Mansa, supervisionado pela Professora e Bióloga Shery Duque Pinheiro e no Zoológico Municipal de Volta Redonda, supervisionado pela Bióloga Natália Teixeira dos Santos. Trabalhando há 4 anos na área de Educação.

E-mail: lidifontes@hotmail.com

\section{Ronald Rodrigues Guimarães}

Bacharel em Medicina Veterinária pela Universidade Federal Rural do Rio de Janeiro e Bacharel em Direito pela Universidade Iguaçu. Especialista em Parasitologia Veterinária (UFRRJ) e em Metodologia do Ensino Superior (UNIG). Mestre em Doenças Parasitárias pela Universidade Iguaçu (2000). Doutor em Saúde Pública pela Escola Nacional de Saúde Pública Sérgio Arouca do Instituto Oswaldo Cruz (2015). Membro do Grupo de Pesquisa Epidemiologia e Biologia Molecular de Agentes Patogênicos e de seus Vetores Biológicos de Importância em Saúde Pública (CNPq). Presidente do Centro de Educação e Pesquisas em Medicina Ambiental (CEMA).

E-mail: ronaldrguimaraes@gmail.com

\section{Introdução e Revisão de Literatura}

A água constitui um dos compostos de maior distribuição e importância do planeta. Sua importância está no fato de que nenhum processo metabólico ocorre sem a sua ação direta ou indireta, portanto ela permeia todos os ciclos biogeoquímicos e assim dinamiza os processos vitais. A formação de grandes centros urbanos, com crescente necessidade de água para o abastecimento doméstico e industrial, além de irrigação e lazer, faz com que, atualmente, a quase totalidade das atividades humanas seja cada vez mais dependente da disponibilidade dos recursos hídricos. Este rápido crescimento populacional e demasiada dependência ao meio ambiente tem sido responsável pelo grande aumento da pressão das atividades antrópicas sobre os recursos naturais.

O biomonitoramento ou, o uso sistemático de respostas biológicas para avaliar mudanças ambientais (LADSON et al., 1999) de corpos hídricos, está cada vez mais sendo usado e aceito como uma importante ferramenta na avaliação da quali- dade da água, revelando o comprometimento de tal ecossistema. Para isso, são usados bioindicadores ou indicadores biológicos, que são organismos que refletem o estado biótico ou abiótico de um meio ambiente, o impacto produzido sobre um habitat, comunidade ou ecossistema, ou também indicar a diversidade de um conjunto de táxons ou biodiversidade de determinada região. Esses organismos são sensíveis a alterações no ambiente ou necessitam de condições específicas para completar alguma fase ou todo seu ciclo de vida. Quando respondem a alterações ambientais, o fazem por meio de reações comportamentais ou metabólicas (ANDREA, 2008).

Uma das comunidades de organismos mais utilizadas em programas de monitoramento biológico são os macroinvertebrados bentônicos (HERING et al., 2004), ou invertebrados visíveis a olho nu que vivem associados ao sedimento. Entre as características que tornam esses organismos bons indicadores das condições ambientais destacam-se a grande diversidade de formas e de habitat, oferecendo um amplo espectro de resposta aos estresses. São de natureza relativamente sedentária, permitindo uma análise espacial eficiente dos efeitos das perturbações. Por viverem em contato com o sedimento, também entram em contato com muitos poluentes, acumulando toxinas a níveis facilmente detectáveis. Seu longo ciclo de vida, comparado a outros organismos, permite a elucidação de mudanças temporais causadas pelas perturbações, além de apresentarem metodologias de análise e coleta simples e de baixo custo, taxonomia conhecida, e boa disponibilidade de chaves de identificação (ROSENBERG et al., 1986). A estrutura da comunidade de macroinvertebrados bentônicos reflete o estado de todo o ecossistema aquático (REICE \& WOHLENBERG, 1993), onde o estabelecimento de espécies nas comunidades bentônicas depende do número de espécies residentes, dos tipos de substratos (BUNN \& DAVIES, 1992; BAPTISTA et al., 2001), dos padrões de corrente do regime de luz e das concentrações de nutrientes dos rios (MALTCHIK \& FLORÍN, 2002). Deste modo, os organismos bentônicos são diretamente afetados pela alteração na composição natural dos sistemas aquáticos, seja diminuindo ou aumentando sua população (MARQUES \& BARBOSA, 2001). Geralmente, há uma diminuição da diversidade da fauna durante processos de contaminação, mostrando uma progressão cronológica, ou seja, esse processo torna-se mais crítico para a comunidade bentônica ao longo do tempo, podendo a fauna ser completamente eliminada do meio. Em casos de poluição severa, esses locais são claramente afetados e a comunidade tende a desenvolver cadeias alimentares mais curtas (GRUMIAUX et al., 1998).

Segundo Goulart et al. (2003), os macroinvertebrados bentônicos podem ser classificados em organismos sensíveis ou intolerantes, que são aqueles típicos de ambientes limpos e de boa qualidade de águas (como ninfas de Plecoptera e larvas de Trichoptera - Insecta); organismos tolerantes ou facultativos (alguns Heteroptera e Odonata - Insecta e Amphipoda - Crustacea); e organismos considerados mais resistentes (Chironomidae - Diptera, Insecta e Oligochaeta - Annelida), de acordo com sua resposta frente às condições do ambiente. A predominância de determinado grupo de organismos no meio pode oferecer indícios sobre as condições da qualidade da água, permitindo avaliar os efeitos da poluição sobre o corpo d'água de maneira holística (MOULTON, 1998).

$\mathrm{O}$ uso de bioindicadores tem sido corrente na avaliação de impactos ambientais provocados pela má administração do ambiente, pois animais, plantas, microrganismos e suas complexas interações com o meio, respondem de maneira diferenciada às modificações da paisagem, produzindo informações, que não só 
indicam a presença de poluentes, mas também como esses interagem com a natureza, proporcionando uma melhor indicação de seus impactos na qualidade dos ecossistemas (SOUZA, 2001).

A dependência do homem moderno dos ecossistemas aquáticos é ainda maior e mais evidente nas regiões altamente industrializadas, nas quais a demanda de água se torna cada vez maior. Além disso, nestas regiões, grande parte dos efluentes domésticos e industriais é lançada diretamente nos corpos d'água, reduzindo ainda mais a possibilidade de utilização dos recursos hídricos (ESTEVES, 1988). Quando o homem através da exploração age sobre os regimes hídricos alteram, em efeito dominó, toda a dinâmica ecológica dos rios, obrigando a natureza a procurar novos padrões de organização desses ecossistemas.

Considerando a importância da comunidade bentônica na estrutura e dinâmica dos ecossistemas aquáticos e seu relevante papel como indicador biológico de estresse ambiental, optou-se pelo estudo desses organismos para avaliar possíveis respostas às alterações ambientais na qualidade da água do rio Paraíba do Sul, em Barra Mansa, estado do Rio de Janeiro.

O Rio Paraíba do Sul possui sua bacia estendida pelo território de três estados - São Paulo, Minas Gerais e Rio de Janeiro - que é considerada uma das três maiores bacias hidrográficas secundárias do Brasil, abrangendo uma área aproximada de $57.000 \mathrm{~km}^{2}$. O rio é formado pela confluência dos rios Paraitinga e Paraibuna, nasce na Serra da Bocaina, no estado de São Paulo, fazendo um percurso total de $1.120 \mathrm{~km}$ até a foz no município de Atafona, no Norte Fluminense. É um rio de jurisdição federal.

A quantificação e/ou qualificação de macroinvertebrados bentônicos ajuda na avaliação da emissão de poluentes que possam causar a alteração dos ecossistemas aquáticos. Em função disto, o presente estudo objetiva:

- Realizar o levantamento de macroinvertebrados no Rio Paraíba do Sul utilizando-os como bioindicadores da qualidade da água no município de Barra Mansa, estado do Rio de Janeiro;

- Contribuir para pesquisas futuras, disponibilizando dados para pesquisadores que estejam se iniciando no uso do biomonitoramento para avaliação da qualidade da água, bem como para estudantes das áreas de limnologia e de recursos hídricos em geral;

- Avaliar a predominância de determinados grupos da comunidade bentônica em função das alterações das condições físicas da água percebidas ao longo do rio;

- Avaliar os resultados obtidos, a fim de verificar a eficácia do estudo em detectar o incremento da poluição no local escolhido.

O grande desafio do biomonitoramento nos sistemas aquáticos é definir um indicador ideal (bioindicador) cuja presença, abundância e/ou comportamento reflitam o efeito de um estressor na biota (BONADA et al., 2006).

A comunidade de macroinvertebrados bentônicos é um importante componente do sedimento de rios e lagos, sendo fundamental para a dinâmica de nutrientes, a transformação de matéria e o fluxo de energia (CALLISTO \& ESTEVES, 1995). Por essas características peculiares, os invertebrados aquáticos são bons sensores de alterações ambientais e, por isso, têm sido cada vez mais utilizados como bioindicadores da qualidade da água (CORBI et al., 2006).

Os invertebrados compreendem o maior número de indivíduos, espécies e biomassa em ambientes lóticos. Eles são representados por diversos filos, como Arthropoda (insetos, ácaros e crustáceos), Mollusca (gastrópodes e bivalves), Annelida (oligoquetas), Nematoda e Platyhelminthes (HAUER \& RESH, 1996). Entre a fauna de invertebrados, os insetos se destacam, já que dominam os ecossistemas aquáticos, sendo sua distribuição relacionada às características morfométricas e físico-químicas do habitat, à disponibilidade de recursos alimentares e ao hábito das espécies (RESH \& ROSENBERG, 1984; BOLTOVSKOY et al., 1995; MERRITT \& CUMMINS, 1996). Os crustáceos e moluscos podem ser abundantes, mas raramente se apresentam com grande diversidade (GULLAN \& CRANSTON, 1996).

Os agentes estressores dos ecossistemas fluviais são de natureza e intensidade variadas e tem papel relevante na destruição e degradação dos habitats. As alterações morfológicas dos rios, além de afetarem o regime da vazão, reduzem o corredor fluvial e degradam a zona ripária com consequentes perdas na biodiversidade e na integridade ecológica desses ambientes (RODRIGUES et al., 2010).

Segundo Pratt \& Coller (1976), a avaliação biológica torna possível a detecção de poluentes devido a capacidade dos organismos reagirem a alterações ambientais modificando suas funções vitais ou sua composição química. Rinaldi (2007) defende ainda que enquanto a avaliação físico-química reflete a condição momentânea do corpo d'água, o monitoramento biológico apresenta um somatório temporal dos fatores ambientais, fornecendo um indicativo das condições passadas e atuais.

A qualidade do hábitat é um dos fatores mais importantes no sucesso de colonização e estabelecimento das comunidades biológicas em ambientes lênticos ou lóticos. A flora e a fauna presentes em um sistema aquático são também influenciadas pelo ambiente físico do corpo d'água (geomorfologia, velocidade da corrente, vazão, tipo de substrato, tempo de retenção) estando estreitamente relacionada às atividades humanas realizadas à sua volta. O primeiro passo para a compreensão de como as comunidades de macroinvertebrados bentônicos estão reagindo à alteração da qualidade de água é identificar quais variáveis físicas e biológicas estão afetando os organismos (TATE \& HEINY, 1995).

As alterações na qualidade de água, resultantes dos processos de evolução natural e de ação antrópica, se manifestam pela redução acentuada da biodiversidade aquática, em função da desestruturação do ambiente físico, químico e alterações na dinâmica e estrutura das comunidades biológicas, sendo que o uso de bioindicadores (espécies, grupos de espécies ou comunidades) permite uma avaliação integrada dos efeitos ecológicos causados por múltiplas fontes de poluição (CALLISTO et al., 2001a, b). Além disso, os bioindicadores complementam as informações sobre qualidade das águas, especialmente para a avaliação de impactos ambientais decorrentes de descargas pontuais de esgotos domésticos e efluentes industriais.

$\mathrm{O}$ primeiro índice a considerar a comunidade biológica para avaliação usando organismos aquáticos e bactérias foi o Sistema Sapróbio desenvolvido por Kolkowitz e Marsson em 1909, considerando o poder indicador de cada organismo (MARGALEF, 1983). Vários outros índices foram desenvolvidos em seguida, como o Índice Biótico de Trent (WOODIWISS, 1964), o Indice Biótico Francês (1968), o Biological Monitoring Working Party System-BMWP(1978 e 1979) entre outros (METCALFE, 1989).

Quando o sistema saprobiótico foi proposto no início do século passado, teve boa aceitação entre os limnólogos europeus, que desenvolveram uma série de estudos que culminaram na elaboração do Trent Biotic Index (Índice Biótico de Trent) - TBI, que se tornou a base da maioria dos índices modernos. O TBI foi desenvolvido considerando tanto a presença quanto a ausência da diversidade de espécies. Chandler (1970) desenvolve, a partir do TBI, o "Chandler's Score System", que por sua vez origina o BMWP, quando o Departamento de Meio Ambiente britânico 
formou, em 1976, um grupo de trabalho, que tinha por objetivo principala elaboração de um sistema de biomonitoramento para os rios da Inglaterra. Este índice sofreu alterações na sua forma e foi adaptado para outros rios, por Alba-Tercedor (1996).

Alba-Tercedor (1996) afirma ainda que os índices de qualidade biológica são ferramentas de primeira grandeza para avaliar a qualidade biológica da água, uma vez que as legislações encontram dificuldades em estabelecer limites sobre as quantidades permitidas de cada contaminante que podem ou não ser toleradas nos efluentes que vertem para os rios.

Um procedimento envolvendo os macroinvertebrados identificados em nível de família (em alguns casos classe ou ordem), utilizando apenas a presença ou ausência destes nas amostras, foi desenvolvido para ser usado no "1980 River Water Quality Survey in Great Britain" (National Water Council, 1981 in Wright, 1995). Mais tarde foi estudado e aplicado também por Armitage et al., (1983), com auxílio do National Water Council. Este índice foi chamado de Biological Monitoring Working Party System (BMWP).

O índice BMWP utiliza os macroinvertebrados aquáticos, mas pode também ser utilizado para os macroinvertebrados bentônicos. Este índice ordena as famílias de macroinvertebrados aquáticos em 9 grupos, segundo um gradiente de menor a maior tolerância dos organismos quanto à poluição orgânica. A cada família se fez corresponder uma pontuação, que oscila de 10 a 1, sendo que as famílias mais sensíveis à contaminação recebem as pontuações maiores, chegando, em ordem decrescente, até 1, onde estão aquelas mais tolerantes (LOYOLA, 2000).

\section{Materiais e Métodos}

O estudo foi realizado no município de Barra Mansa, situado ao sul do estado do Rio de Janeiro, às margens do Rio Paraíba do Sul, onde foi realizado o levantamento de macroinvertebrados bentônicos, para identificação do impacto ambiental causado por ações antrópicas. Esse município fica localizado em uma microrregião do Médio Vale do Paraíba dentro da mesorregião do Sul Fluminense, entre as Serras do Mar e da Mantiqueira (22 $\left.32^{\prime} 39^{\prime \prime S}, 4^{\circ} 10^{\prime} 17^{\prime} \mathrm{O}\right)$, altitude de $381 \mathrm{~m}$, com ponto culminante a $1.305 \mathrm{~m}$, na Serra do Rio Bonito (contrafortes da Serra da Mantiqueira), no Distrito de Nossa Senhora do Amparo. Possui uma área de $548,9 \mathrm{~km}^{2}$ e população estimada de 179.697 habitantes (IBGE, 2010). Os municípios limítrofes são ao Norte, Valença e Quatis; ao Sul, Rio Claro, Piraí e Bananal (SP); a Leste, Volta Redonda, Barra do Piraí e Piraí; e a Oeste, Resende, Quatis e Porto Real.

O clima de Barra Mansa é mesotérmico, com verões quentes e chuvosos e inverno seco. A umidade relativa do ar é de $77 \%$ e a temperatura média mínima anual é de $16^{\circ} \mathrm{C}$ e a média máxima anual é de $28^{\circ} \mathrm{C}$. O período de chuvas está entre os meses de novembro e março, com pluviosidade de aproximadamente 1.380 $\mathrm{mm}$ /ano (IBGE, 2010).

O Rio Paraíba do Sul, participa da estrutura hidrográfica do município de Barra Mansa, drenando vasta região por meio de uma grande quantidade de rios e córregos tributários. Pela margem direita, os principais afluentes são o Rio Bananal, Rio Barra Mansa (que tem sua nascente no município de Rio Claro), Rio Bocaina, Ribeirão Brandão e o córrego Cotiara; pela margem esquerda, Rio Turvo e os córregos Ano Bom e Água Comprida.

\section{Pontos de Coleta}

Foram selecionados, ao longo da margem direita do Rio Paraíba do Sul, sete pontos para coleta de macroinvertebrados bentônicos, com distância de $20 \mathrm{~m}$ entre cada ponto. Em cada local foram realizadas três coletas, nos meses de fevereiro, maio e outubro de 2014.

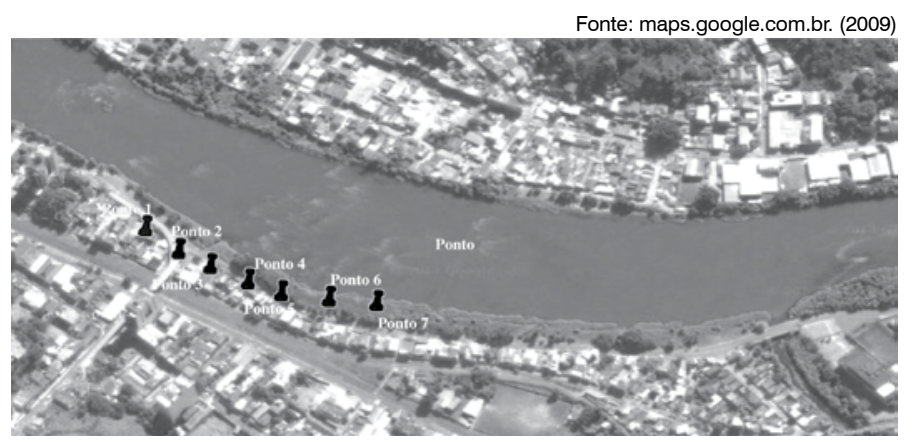

Figura 1: Pontos de coleta de macroinvertebrados bentônicos no Rio Paraíba do Sul nos meses de fevereiro, maio e outubro de 2014, Barra Mansa, Rio de Janeiro, Brasil

Fonte: maps.google.com.br. (2009)

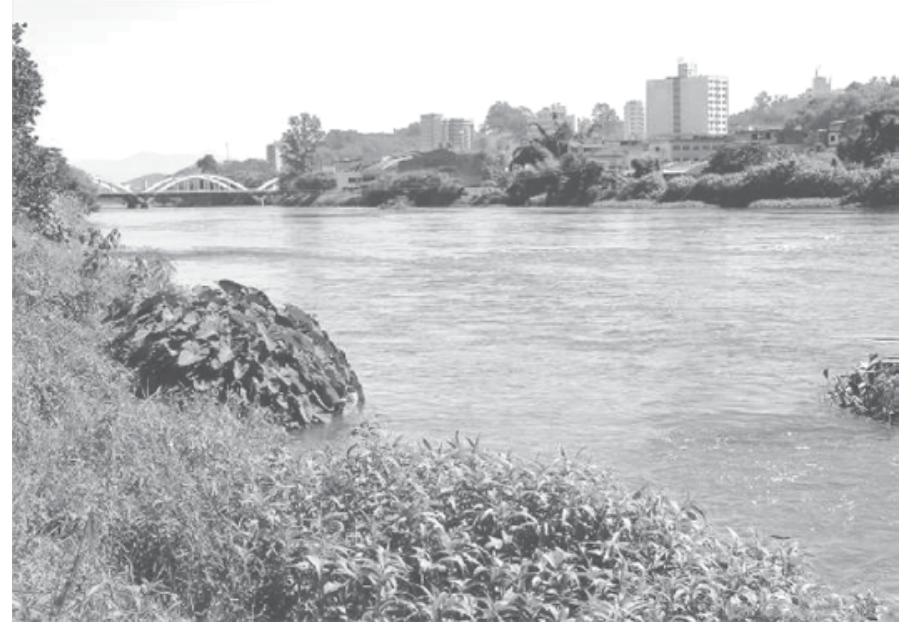

Figura 2: Vista parcial do Rio Paraíba do Sul com estreita faixa de mata ciliar nas margens direita e esquerda, Barra Mansa, Rio de Janeiro, Brasil. Foto: Lidiane Fontes de Carvalho Silva

As coletas foram realizadas por meio de puçá com rede em forma de $\mathrm{D}$, sendo arrastado contra a corrente por cerca de 1 metro de extensão sobre sedimento arenoso. $\mathrm{O}$ material coletado foi armazenado em recipientes de plástico de 250 e $500 \mathrm{~mL}$, contendo água do local, e posteriormente acondicionado em caixas de isopor de 5l. Todos os recipientes foram identificados com etiquetas contendo a data, ponto de coleta e número da coleta. Além da coleta de macroinvertebrados bentônicos, foi aferida a temperatura da água em cada ponto e a profundidade do local de realização do arraste.

Após a coleta, todo o material foi encaminhado ao laboratório de Zoologia do Centro Universitário de Barra Mansa, onde se procederam às etapas de lavagem, flutuação (ou pré-triagem), triagem e identificação dos organismos. Na primeira etapa, o material coletado foi lavado em água corrente e os elementos grosseiros do particulado foram separados, de modo a facilitar a triagem posterior dos macroinvertebrados. Após essa etapa, foi realizado o processo de flutuação, onde o particulado separado foi disposto em bandejas de polietileno translúcidas contendo uma solução supersaturada de açúcar (1 litro de água para 250 
gramo de açúcar). Nesse procedimento, os macroinvertebrados flutuam, facilitando e otimizando a etapa de identificação.

Com auxílio de uma pinça de aço inoxidável e uma lupa manual, os macroinvertebrados foram retirados das bandejas e colocados em novos recipientes de plástico de 250 e $500 \mathrm{~mL}$ contendo álcool etílico hidratado a $70 \%$ para a rápida fixação e preservação, conforme Tomm (2001). Os referidos recipientes foram etiquetados e agrupados de acordo com os pontos de coleta.

Após a triagem, procedeu-se à identificação dos organismos fixados. Os macroinvertebrados foram dispostos em placas de petri e examinados por meio de microscópio estereoscópico, e classificados até o nível de família, utilizando-se as chaves disponíveis na literatura (ANGRISANO, 1995; WIGGINS, 1996; CARVALHO \& CALIL, 2000; COSTA et al., 2004; LOPRETTO \& TELL, 1995; COURTNEY \& MERRITT, 2008).

Para avaliação e classificação da qualidade da água com relação à fauna de macroinvertebrados bentônicos foi aplicado o índice biológico BMWP, conforme tabelas 1 e 2 .

Tabela 1

Pontuação das famílias de macroinvertebrados bentônicos para a obtenção do índice BMWP

\begin{tabular}{|c|c|}
\hline FAMÍLIAS & PONTUAÇÃO \\
\hline $\begin{array}{l}\text { Siphlonuridae, Heptageniidae, Leptophlebiidae, Potamanthidae, Ephemeridae (Efemerópteros); } \\
\text { Taeniopterygidae, Leuctridae, Capniidae, Perlodidae, Perlidae, Chloroperlidae (Plecópteros); } \\
\text { Aphelocheiridae, Phryganeidae, Molannidae, Beraeidae, Odontoceridae, Leptoceridae, Goeridae, } \\
\text { Lepidostomatidae, Brachycentridae, Sericostomatidae (Tricópteros); Athericidae, Blephariceridae (Dípteros). }\end{array}$ & 10 \\
\hline $\begin{array}{l}\text { Astacidae (Crustáceos); Lestidae, Calopterygidae, Gomphidae, Cordulegastridae, Aeshnidae, Corduliidae, } \\
\text { Libellulidae (Odonatas); Psychomyiidae, Philopotamidae, Glossosomatidae (Tricópteros). }\end{array}$ & 8 \\
\hline $\begin{array}{l}\text { Ephemerellidae (Efemerópteros); Nemouridae (Plecópteros); Rhyacophilidae, Polycentropodidae, } \\
\text { Limnephilidae (Tricópteros). }\end{array}$ & 7 \\
\hline $\begin{array}{l}\text { Neritidae, Viviparidae, Ancylidae, Unionidae, Corophiidae, Gammaridae, Ampullaridae, Thiaridae } \\
\text { (Moluscos); Hydroptilidae, (Tricópteros); Platycnemididae, Coenagrionidae (Odonatas). }\end{array}$ & 6 \\
\hline $\begin{array}{l}\text { Oligoneuridae (Efemerópteros); Dryopidae, Elmidae, Helophoridae, Hydrochidae, Hydraenidae, } \\
\text { Clambidae (Coleópteros); Hydropsychidae (Tricópteros); Tipulidae, Simuliidae, (Dípteros); Planariidae, } \\
\text { Dendrocoelidae, Dugesiidae (Turbelárias). }\end{array}$ & 5 \\
\hline $\begin{array}{l}\text { Baetidae, Caenidae (Efemerópteros); Haliplidae, Curculionidae, Chrysomelidae (Coleópteros); Tabanidae, } \\
\text { Stratiomyiidae, Empididae, Dolichopodidae, Dixidae, Ceratopogonidae, Psychodidae, Anthomyidae, } \\
\text { Limoniidae (Dípteros); Sialidae (Megalópteros); Piscicolidae (Hirudíneas); Hidracarina (Ácaros). }\end{array}$ & 4 \\
\hline $\begin{array}{l}\text { Mesoveliidae, Hydrometridae, Gerridae, Nepidae, Naucoridae, Pleidae, Notonectidae, Corixidae } \\
\text { (Hemípteros); Helodidae, Hydrophilidae, Hygrobiidae, Dytiscidae, Gyrinidae (Coleópteros); Valvatidae, } \\
\text { Hydrobiidae, Lymnaeidae, Physidae, Planorbidae, Bithyniidae, Bythinellidae, Sphaeriidae (Moluscos); } \\
\text { Glossiphoniidae, Hirudidae, Erpobdellidae (Hirudíneas); Asellidae, Ostracoda (Crustáceos). }\end{array}$ & 3 \\
\hline Chironomidae, Culicidae, Muscidae, Thaumaleidae, Ephydridae (Dípteros). & 2 \\
\hline Subclasse Oligochaeta (Todas as famílias). & 1 \\
\hline
\end{tabular}

Fonte: Adaptado de Brigante et al. (2003)

Tabela 2 - Classes de qualidade e significado dos valores do índice BMWP

\begin{tabular}{c|c|c|c}
\hline CLASSE & QUALIDADE & VALOR & SIGNIFICADO \\
\hline I & BOA & $>150$ & Águas muito limpas \\
\hline II & $101-120$ & & $\begin{array}{c}\text { Águas limpas, sem alteração ou } \\
\text { contaminação evidente }\end{array}$ \\
\hline III & ACEITÁVEL & $61-100$ & Águas com algum sinal de contaminação \\
\hline IV & DUVIDOSA & $36-60$ & Águas contaminadas \\
V & CRÍTICA & $16-35$ & Águas muito contaminadas \\
\hline
\end{tabular}




\section{Resultados e Discussão}

O Rio Paraíba do Sul apresenta uma estreita faixa de mata ciliar, demonstrando sinais de impacto ambiental. Além deste fato, ao longo do trecho avaliado, foram identificados três pontos diretos de descarga de efluentes domésticos (pontos 1, 3 e 7), contribuindo para a degradação da qualidade de suas águas. A temperatura das águas nos pontos de coleta variou entre $25^{\circ} \mathrm{C}$ e $28^{\circ} \mathrm{C}$ e a profundidade do arraste entre 1,07 e 1,25, conforme Tabela 3 .

Tabela 3

Data de coletas, temperaturas aferidas e profundidades nos diferentes pontos de coleta no Rio de Paraíba do Sul, Barra Mansa, Rio de Janeiro, Brasil

\begin{tabular}{c|c|c|c}
$\begin{array}{c}\text { Pontos } \\
\text { de Coleta }\end{array}$ & $\begin{array}{c}\text { Data } \\
\text { da Coleta }\end{array}$ & Temperatura & $\begin{array}{c}\text { Profundidade } \\
\text { do arraste }\end{array}$ \\
\hline Ponto 1 & $21 / 02 / 2014$ & $26^{\circ} \mathrm{C}$ & $1,22 \mathrm{~m}$ \\
Ponto 2 & $21 / 02 / 2014$ & $25^{\circ} \mathrm{C}$ & $1,12 \mathrm{~m}$ \\
Ponto 3 & $01 / 05 / 2014$ & $28^{\circ} \mathrm{C}$ & $1,07 \mathrm{~m}$ \\
Ponto 4 & $01 / 05 / 2014$ & $25^{\circ} \mathrm{C}$ & $1,25 \mathrm{~m}$ \\
Ponto 5 & $02 / 05 / 2014$ & $26^{\circ} \mathrm{C}$ & $1,24 \mathrm{~m}$ \\
Ponto 6 & $15 / 10 / 2014$ & $28^{\circ} \mathrm{C}$ & $1,18 \mathrm{~m}$ \\
Ponto 7 & $15 / 10 / 2014$ & $25^{\circ} \mathrm{C}$ & $1,21 \mathrm{~m}$ \\
\hline
\end{tabular}

Estrutura e composição dos macroinvertebrados bentônicos Foram identificados 211 espécimes (Tabela 4) pertencentes a 11 famílias de macroinvertebrados bentônicos (Tabela 5), distribuídos em três grandes filos: Annelida (10\%) representado por espécies da Classe Clitellata (Subclasse Hirudinea), Arthropoda $(18 \%)$ representado pelos Insecta das ordens Coleoptera, Odonata, Diptera, e Mollusca (72\%) representado pelos Gastropoda.
Tabela 5

Número de pontos atribuído a cada família de macroinvertebrados de acordo com o índice BMWP para análise da qualidade de água, utilizado para análise no estudo

\begin{tabular}{|c|c|}
\hline Táxon & Pontuação \\
\hline Gastropoda & \\
Planorbidae & 03 pontos \\
Physidae & 03 pontos \\
Ampullaridae & 06 pontos \\
Thiaridae & 06 pontos \\
\hline Odonata & \\
Calopterygidae & 08 pontos \\
Gomphidae & 08 pontos \\
\hline Diptera & \\
Chironomidae & 02 pontos \\
Culicidae & 02 pontos \\
\hline Coleoptera & \\
Dytiscidae & 03 pontos \\
\hline Hirudinea & $\mathbf{4 7}$ pontos \\
Glossiphoniidae & \\
Erpobdellidae & 03 pontos \\
\hline Pontuação Total & \\
\hline
\end{tabular}

Tabela 4

Distribuição das famílias de macroinvertebrados bentônicos encontradas nos sete pontos de coleta entre outubro de 2013 e outubro de 2014 no Rio Paraíba do Sul, Barra Mansa, Rio de Janeiro, Brasil.

\begin{tabular}{|c|c|c|c|c|c|c|c|c|}
\hline Taxon & Ponto1 & Ponto 2 & Ponto 3 & Ponto 4 & Ponto 5 & Ponto 6 & Ponto 7 & Total \\
\hline $\begin{array}{l}\text { Classe Gastropoda } \\
\text { Planorbidae } \\
\text { Physidae } \\
\text { Ampullaridae } \\
\text { Thiaridae }\end{array}$ & $\begin{array}{c}1 \\
15 \\
- \\
1\end{array}$ & $\begin{array}{l}- \\
16 \\
- \\
-\end{array}$ & $\begin{array}{l}2 \\
1 \\
2 \\
-\end{array}$ & $\begin{array}{l}- \\
83 \\
- \\
-\end{array}$ & $\begin{array}{l}- \\
15 \\
- \\
9\end{array}$ & $\begin{array}{l}- \\
4 \\
1 \\
-\end{array}$ & $\begin{array}{l}- \\
1 \\
1 \\
-\end{array}$ & $\begin{array}{c}3 \\
135 \\
4 \\
10\end{array}$ \\
\hline $\begin{array}{l}\text { Classe Insecta } \\
\text { Ordem Odonata } \\
\text { Calopterygidae } \\
\text { Gomphidae } \\
\text { Ordem Diptera } \\
\text { Chironomidae } \\
\text { Culicidae } \\
\text { Ordem Coleoptera } \\
\text { Dytiscidae }\end{array}$ & $\begin{array}{l}- \\
2 \\
- \\
2 \\
-\end{array}$ & $\begin{array}{l}1 \\
- \\
- \\
- \\
-\end{array}$ & $\begin{array}{l}3 \\
4 \\
- \\
2 \\
1\end{array}$ & $\begin{array}{l}3 \\
1 \\
- \\
1\end{array}$ & $\begin{array}{l}5 \\
- \\
- \\
1 \\
1\end{array}$ & $\begin{array}{l}- \\
- \\
2 \\
1 \\
-\end{array}$ & $\begin{array}{l}2 \\
- \\
4 \\
1\end{array}$ & $\begin{array}{c}14 \\
7 \\
6 \\
8 \\
2\end{array}$ \\
\hline $\begin{array}{l}\text { Classe Clitellata } \\
\text { Hirudinea } \\
\text { Glossiphoniidae } \\
\text { Erpobdellidae }\end{array}$ & $\begin{array}{l}1 \\
-\end{array}$ & - & - & $\begin{array}{l}2 \\
-\end{array}$ & $\begin{array}{l}2 \\
-\end{array}$ & - & - & $\begin{array}{c}5 \\
17\end{array}$ \\
\hline TOTAL & 22 & 17 & 15 & 90 & 33 & 21 & 13 & 211 \\
\hline
\end{tabular}


No geral, os pontos de coleta 4 e 5 apresentaram maior abundância de espécies de macroinvertebrados bentônicos amostradas, respectivamente, $42,6 \%$ e $15,6 \%$. A classe Gastropoda foi dominante, com a presença de $72 \%$ do total de organismos e a ordem Hirudinea foi relativamente expressiva, com 10,4\%. A família Physidae foi a que apresentou exemplares em todos os pontos de coleta e a Dytiscidae, apresentou apenas 2 indivíduos, sendo um no ponto 3 e outro no ponto 5 . Todos os pontos apresentaram uma baixa diversidade de famílias, com pouca diferença entre eles, provavelmente devido à situação do meio, onde efluentes domésticos são depositados, determinando grande poluição do rio. Através de observação local, foi possível notar que as famílias encontradas são condizentes com a situação observada, o que demonstra a importância de áreas verdes e mata ciliar preservadas, para a qualidade da água e, consequentemente, para todo o ecossistema. De acordo com Merritt e Lawson (1992), a produção primária oriunda da mata ciliar é uma importante fonte de alimentação e proporciona diversos habitats para inúmeras espécies aquáticas.

\section{Índice BMWP}

A pontuação para o índice BMWP para o trecho do Rio Paraíba do Sul analisado no período do estudo, foi de " 47 ", classificando a qualidade da água como "duvidosa" (águas contaminadas). A baixa diversidade de famílias encontradas e a ausência de alguns grupos de organismos sensíveis a alterações ambientais indicam que o ambiente estudado apresenta certo grau de degradação, fato este, causado pela grande quantidade de matéria orgânica em decomposição, levando à baixa oxigenação da água. Este impacto reflete a ação antrópica sobre o ambiente aquático, pois o Rio Paraíba do Sul acaba se tornando um grande depósito de efluentes domésticos, poluição urbana e poluição industrial.

\section{Considerações finais}

Entre os problemas ambientais que afetam a qualidade das águas do Rio Paraíba do Sul, destacam-se, predominantemente, os problemas relativos à poluição industrial, ao esgotamento sanitário e à erosão.

Para a melhoria da qualidade das águas do Rio Paraíba do Sul deve-se priorizar investimentos para o desenvolvimento e implementação de programas eficazes de monitoramento permanente deste Rio.

A gestão dos ecossistemas aquáticos é, portanto, cada vez mais um tema preponderante, tendo entre outras finalidades, a de acompanhar as condições e a disponibilidade desses recursos, definindo seus usos e propondo melhorias.

\section{REFERÊNCIAS}

ALBA-TERCEDOR, J. Macroinvertebrados acuáticos y calidad de las aguas de los rios. In. IX Simpósio del Agua en Andalucia (SIAGA), Almeria, pp. 203-213. 1996.

ANDRÉA, M.M. Bioindicadores ecotoxicológicos de agrotóxicos. (Comunicado Técnico, 83). Disponível em: <http://www.biologico. sp.gov.br/artigos_ok.php?id_artigo $=83 \#>$. Acesso em: fevereiro de 2014

ANGRISANO, E.B. Insecta Trichoptera, p. 1199-1237. In: E. C. Lopretto \& G. Tell (Eds.). Ecosistemas de aguas continentales, metodologia para su estudio. Tomo III. La Plata, Ediciones Sur, 1401 p. 1995.

ARMITAGE P.D.; MOSS D.; WRIGHT J.F. \& FURSE M.T. The performance of a new biological water quality score system based on macroinvertebrates over a wide range of unpolluted running water sites. Water Research 17, 333-347.1983.

BAPTISTA, D.F.; DORVILLÉ, L.F.M.; BUSS, D.F. \& NESSIAMIAN, J.L. Spatial and temporal organization of aquatic insects assemblages in the longitudinal gradient of a tropical river. Revista Brasileira de Biologia, Rio de Janeiro, 61 (2): 295-304. 2001.

BOLTOVSKOY, D. \&. JANKILEVICH, S.S. Radiolarian distribution in east equatorial Pacific plankton. Oceanol. Acta, 8: 101- 123. 1985. BONADA, N.; PRAT, N.; RESH, V.H. \& STATZNER, B. Developments in aquatic insect biomonitoring: a comparative analysis of recent approaches. Annual Review of Entomology ,51, 495-523. 2006. BRIGANTE, J.; ESPÍNDOLA, E.L.G.; POVINELI, J.; NOGUEIRA, A.M. \& MORRAYE, M.A. Comunidade de Macroinvertebrados bentônicos no Rio Mogui-Guaçu. In: Brigante, J.; Espíndola, E. L. G.(ed.) Limnologia Fluvial-Estudo no Rio Mogi-Guaçu. Ed. RiMa.255p. 2003.

BUNN, S.E. \& DAVIES, P.M. Community structure of macroinvertebrate fauna and water quality of saline river system in south-western Australia. Hydrobiologia, 248: 143-160. 1992.

CALliSTO, M. \& ESTEVES F. A. Distribuição da comunidade de macroinvertebrados bentônicos em um lago amazônico impactado por rejeito de bauxita. Lago Batata (Para, Brasil). Oecologia Brasiliensis, 1: 335-348. 1995.

CALLISTO, M.; MORETTI, M. \& GOULART, M. Macroinvertebrados bentônicos como ferramenta para avaliar a saúde de riachos. Revista Brasileira de Recursos Hídricos, Porto Alegre, 6 (1): 71-82. 2001a.

CALlistO, M.; MORENO, P. \& BARBOSA, F.A.R. Habitat diversity and benthic functional trophic groups at Serra do Cipó, Southeast Brazil. Revista Brasileira de Biologia, Rio de Janeiro, 61 (2): 259-266. 2001b.

CARVALHO, A.L.\& CALIL, E.R. Chaves de identificação para as famílias de Odonata (Insecta) ocorrentes no Brasil, adultos e larvas. Papéis Avulsos de Zoologia, S. Paulo, 41 (15):223-241. 2000.

IBGE. Censo Populacional 2010. Instituto Brasileiro de Geografia e Estatística (IBGE). Disponível em: http://censo2010.ibge.gov. br/. 2010.

CHANDLER, J.R. A biological approach to water quality management. Water Pollution Control, 69: 415-421. 1970.

CORBI, J. J.; TRIVINHO-STRIXINO, S.; DOS SANTOS, A. \& DEL GRANDE, M. Environmental diagnostic of metals and organochlorated compounds in streams near sugar cane plantations activity (State of São Paulo, Brazil). Quimica Nova, 29: 61-65. 2006.

COSTA, J.M.; SOUZA, L.O.; OLDRINI, I. \& BOTELHO, B. Chave para identificação das famílias e gêneros das larvas de conhecidas de Odonata do Brasil: Comentários e registros bibliográficos (Insecta, Odonata). Publicações avulsas do Museu Nacional. Rio de Janeiro, 99: 1-44. 2004.

COURTNEY, G.W. \& MERRITT, R.W. Aquatic Diptera. Part one. Larvae of aquatic Diptera, Capítulo 22, pp. 687-722. In R.W. Merritt, K.W. Cummins \& M.B. Berg (eds.). An Introduction to the Aquatic Insects of North America. 4a edição. Kendall/Hunt Publishing Co. Dubuque, Iowa. 1158 p. 2008.

ESTEVES, F.A. Fundamentos de Limnologia. 2. ed. Rio de Janeiro: Interciência, 1988.

GOOGLE MAPS. Barra Mansa RJ. Disponível em: < http://maps. google.com.br/maps>. Acesso em: 20 Out. 2014.

GOULART, M.D.C.; CALLISTO, M. 2003. Bioindicadores de Qualidade de Água como Ferramenta em Estudos de Impacto Ambiental. Revista da FAPAM. Minas Gerais, ano 2, n. 01, 2003.

GRUMIAUX, F.; LEPRÊTRE, A. \& DHAINAUT-COURTOIS, N. Effect of sediment quality on benthic macroinvertebrate communities in streams in the north of France. Hydrobiologia, The Hague, 385(1-3): 33-46. 1998. 
GULLAN, P.J. \& CRANSTON, P.S. The insects: an outline of entomology. London: Chapmam \& Hall. 113p. 1996.

HAUER, F.R. \& V.H. RESH. Benthic macroinvertebrates, p. 339369. In: F.R. HAUER \& G.A. LAMBERTI (Eds). Stream ecology. San Diego, Academic Press, 674p. 1996.

HERING, D.; BOHMER, R.; HAASE, P. \& SCHAUMBURG, J. 2004. Asssessing stream in Germany with benthuc invertebrates: selection of candidate metrics. Limnologica 34: 398-415. 2004.

LADSON A. R.; WHITE, L.J.; DOOLAN, J.A.; FINLAYSON, B.L.; HART, B.T.; LAKE, P.S. \& TILLEARD, J.W. Development and testing of an Index of Stream Condition for waterway management in Australia. Freshwater Biology, 41: 453-469. 1999.

LOPRETTO, E.C. \& TELL, G. Ecosistemas de aguas continentales: metodologías para su estudio. vol. 1, 2 e 3. Ediciones Sur, La Plata. 1995.

LOYOLA, R.G.N. Atual estágio do IAP no uso de indices biológicos de qualidade. In: Anais do V Simpósio de Ecossistemas Brasileiros: Conservação. UFES, Vitória - ES. v. 1, p.46-52. 2000.

MALTCHIK, L. \& FLORIN, M. Perspectives of hydrological disturbance as the driving force of Brazilian semiarid stream ecosystems. Acta Limnologica Brasiliensia, 14(3): 35-41. 2002.

MARGALEF, R. Limnologia. Editora Omega, Barcelona, 1100 p. 1983. MARQUES, M.M. \& BARBOSA, F. Biological quality of waters from an impacted tropical watershed (middle Rio Doce basin, southeast Brazil), using benthic macroinvertebrate communities as an indicator. Hydrobiologia, 457:69-76. 2001.

MERRITT, R.W. \& LAWSON, D. The role of leaf litter macroinvertebrates in stream-floodplain dynamics. Hydrobiology, 248: 65-77. 1992.

MERRITT, R.W. \& CUMMINS, K.W. An introduction to the aquatic insects of North America. Dubuque, Kendall/Hunt, $3^{\text {rd }}$ ed., 722p. 1996.

METCALFE, J.L. Biological water quality assessment of running waters based on macroinvertebrate communities: history and present status in Europe. Environmental Pollution, 139: 60-101. 1989.

MOULTON, T.P. Saúde e integridade do ecossistema e o papel dos insetos aquáticos. In: Nessimian, J.L. A.L. Carvalho (Eds.). Series Oecologia Brasiliensis, vol. V. PPGE-UFRJ. Rio de Janeiro, Brasil. p. 281-298. 1998.

NATIONAL WATER COUNCIL. River Quality: The 1980 Survey and Future Outlook. London: National Water Council. 1981.

PRATT, J. M. \& COLER, R. A. A procedure for the routine biological evaluation of urban runoff in small rivers. Water Research, 10: 1019-1025. 1976.

REICE, S.R. \& WOHLENBERG, M. Monitoring freshwater benthic macroinvertebrates and benthic processes: Measures for assessment of ecosystem health. In: Freshwater biomonitoring and benthic macroinvertebrates. (Eds. Resh V. H. \& Rosenberg D. M.) 1st edition Chapman \& hall. p. 287-305. 1993

RESH, V.H. \& ROSENBERG, D. M. (Eds). The ecology of aquatic insects. Praeger Scientific, NY. 625 pp. 1984.

RINALDI, S.A. Uso de macroinvertebrados bentônicos na avaliação do impacto antropogênico às margens do Parque Estadual do Jaraguá, São Paulo-SP. Dissertação de Mestrado, Departamento de ecologia da USP, São Paulo. 2007.

RODRIGUES, R.R.; GANDOLFI, S.; NAVE, A.G.; ARONSON, J.; BARRETO, T.E.; VIDAL, C.Y. \& BRANCALION, P.H.S. Large-scale ecological restoration of high-diversity tropical forests in SE Brazil. Forest Ecology and Management, 261(10): 1605-1613. 2010. ROSENBERG, D.M.; HANKS, H.V. \& LEHMKUHL, D.M. Importance of insects in environmental impact assessment. Environmental Management 10, 773 \pm 783.1986.

SOUZA, M.R. A Implantação do Sistema de Gestão Ambiental Se- gundo ISO 14001. Dissertação de Mestrado do programa da Escola Federal de Engenharia de Itajubá. 2001.

TATE, C.M. \& HEINY, J.S. The ordination of benthic invertebrate communities in the South Platte River Basin in relation to environmental factors. Freshwater Biology, 33: 439-454. 1995.

TOMM, I. Avaliação da qualidade da água do rio Toledo (ToledoParaná) através de macroinvertebrados bentônicos. Florianópolis: UFSC, 2001. 213p. Dissertação Mestrado.

WIGGINS, G.B. Larvae of the North American Caddisfly genera (Trichoptera). 2nd Ed. Toronto, University of Toronto Press Incorporated, 457 p. 1996.

WOODIWISS, F.S. A biological system to stream classification used by Trent River Board. Chemy. Ind., 11: 443-447. 1964.

WRIGHT, J.F. Development and use of a system for predicting macroinvertebrates in flowing waters. Australian Journal of Ecology 20: 181-197. 1995. 